\title{
TREMATÓDEOS MONOGENÉTICOS (POLISTOMA- TAS) DA COSTA BRASILEIRA I. SOBRE LOIMOS SCITULUS SP. N. (LOIMOIDAE) E TAGIA ECUADORI (MESERVE, 1938) SPROSTON, 1946 (DICLIDOPHORIDAE) ${ }^{1}$
}

\author{
URSULA BUHRNHEIM \\ Instituto Oswaldo Cruz, Rio de Janeiro, Guanabara \\ (Com 6 figuras)
}

SUMáRIo: Em continuação aos estudos dos trematódeos monogenéticos da Coleção Helmintológica do Instituto Oswaldo Cruz, descrevemos no presente trabalho uma espécie do gênero Loimos MacCallum, 1917, Loimolinae Price, 1936, Loimoidae Bychowsky, 1957 que consideramos nova para a ciência, e assinalamos nova ocorrência de Tagia ecuadori (Meserve, 1938) Sproston, 1946, Tagiinae Yamaguti, 1963, Diclidophoridae Najibina \& Obonikova, 1971, no Atlântico Sul.

Loimos scitulus sp. n. diferencia-se das outras espécies do gênero pelos seguintes caracteres: forma, e estrutura do proaptor, oótipo grande, número de testículos, posição do poro genital, filamento do ovo e forma do opistaptor. Dentre as diferenças dadas Loimos scitulus sp. $\mathrm{n}$. aproxima-se de L. salpinggoides pela estrutura do proaptor, de L. secundus pela posição do poro genital; de $L$. winteri pelo opistaptor.

Quanto aos exemplares de Tagia ecuadori por nós estudados, apesar de ter sido evidenciada uma vagina, identificamos a esta espécie, por apresentar estruturas e medidas que se enquadram nas variações dadas pelos estudiosos do grupo.

PRATICAMENTE a fauna de trema-
tódeos monogenéticos do Atlântico Sul (Costa do Brasil) ainda é desconhecida, embora tenhamos tido a oportunidade de iniciar os estudos sobre alguns respresentantes do grupo que ocorrem nesta região.

Baseados nisso, tentamos com esta publicação ampliar o conhecimento destes helmintos.

\section{MATERIAL E MÉTODOS}

O material estudado é proveniente da Coleção Helmintológica do Instituto Oswaldo Cruz. Foi coletado pelos Kohn, A., Motta, C. S. \& Santos, W. em 1963. Os trematódeos monogenéticos estavam conservados em formol e foram examinados depois de corados com carmim acético, diafanizados com formol e creosoto de Faia e fixados com bálsamo do Canadá.

1 Recebido para publicação a 22-9-71.

(Trabalho do Instituto Oswaldo Cruz, realizado com auxílio do Conselho Nacional de Pesquisas). 


\section{RESULTADOS}

Os resultados obtidos do estudo deste material foram os seguintes:

\section{Loimos scitulus sp. n.}

Morfologia externa: Animais pequenos de corpo fusiforme com a parte posterior em forma de disco (opistaptor). Medem 1,56-1,98 $\mathrm{mm}$ de comprimento e 0,36-0,42 $\mathrm{mm}$ de largura máxima na altura do ovário (fig. 1). A extremidade anterior do corpo apresenta uma abertura terminal grande, que ocupa quase toda a largura. Esta abertura forma uma cavidade de paredes musculares, com 0,060-0,093 $\mathrm{mm}$ de profundidade. Na base desta cavidade abre-se a boca grande e circular. Na cavidade, ainda, encontram-se quatro ventosas pré-orais de tamanhos diversos, 10calizadas uma ao lado da outra, sendo que a maior mede $0,048-0,060 \mathrm{~mm}$ de comprimento por $0,060-0,066 \mathrm{~mm}$ de largura, e encontra-se em uma das extremidades; as três restantes estão dispostas em ordem crescente, de maneira que a maior dessas ocupa a outra extremidade. As suas medidas são respectivamente: $0,024-0,030 \times$ 0,027-0,033 mm;0,027-0,030 × 0,030-0,033 mm; 0,030-0,036 $\times$ 0,033-0,039 $\mathrm{mm}$ de comprimento por largura (fig. 3). O poro genital abre-se logo abaixo da cavidade do proaptor, na região da pré-faringe, a 0,090-0,0150 $\mathrm{mm}$ de extremidade anterior. A abertura da vagina, encontra-se na zona da bifurcação intestinal, deslocada para o lado, a 0,365-0,435 $\mathrm{mm}$ da extremidade anterior. O opistaptor em forma de disco mede $0,450 \mathrm{~mm}$ de largura e tem uma margem membranosa, na qual se encontram aproximadamente 16 microganchos. Existem dois microganchos situados no bordo posterior do opistaptor, implantados na sua musculatura, que medem 0,033-0,042 $\mathrm{mm}$ de comprimento (fig. 4). A musculatura é forte, radial e dirige-se para o centro do opistaptor formando sulcos ordenados. Apresenta ainda dois pares de órgãos de fixação elíticos glandulares e musculares, que medem 0,033 a $0,042 \mathrm{~mm}$ de comprimento por $0,096-0,120 \mathrm{~mm}$ de largura, localizados na região central do opistaptor.
Aparelho digestivo: Abertura b u c a l bem desenvolvida de paredes grossas, musculares, que se abre no fundo da cavidade do proaptor. Pré-faringe de paredes delgadas presentes. Faringe de 0,150-0,165 mm de comprimento por $0,129-0,150 \mathrm{~mm}$ de largura. Esta faringe é formada por duas partes: uma anterior, menos musculosa, em forma de colarinho medindo 0,033-0,036 $\mathrm{mm}$ de comprimento por 0,096-0,099 $\mathrm{mm}$ de largura; outra posterior esférica, mais volumosa, de musculatura bem desenvolvida formando feixes. Esôfago curto. Cecos intestinais não ramificados, que se estendem até a extremidade posterior do corpo.

\section{Aparelho reprodutor (Fig. 5)}

Aparelho genital feminino: O vári o lobado, pré-testicular, cecal e intercecal, situado no segundo quarto do corpò, ocupando um campo de 0,135-0,153 $\mathrm{mm}$ de comprimento por $0,180-0,273 \mathrm{~mm}$ de largura. Oviduto curto ao qual se juntam os vitelodutos e o conduto vaginal, formando então o oótipo em forma de pera, que mede $0,105-0,138 \mathrm{~mm}$ de comprimento por 0,036-0,078 $\mathrm{mm}$ de largura. Daí parte o útero reto, pouco visível, que se abre no poro genital; em alguns exemplares contendo um ovo de $0,105-0,111 \mathrm{~mm}$ de comprimento por 0,054-0,072 $\mathrm{mm}$ de largura. Ovos com filamento de $0,120-0,138 \mathrm{~mm}$ de comprimento. Poro vaginal abre-se látero-ventralmente ao nível da bifurcação intestinal; em seguida alarga-se formando uma dilatação (espermateca), que mede 0,096-0,135 $\mathrm{mm}$ de comprimento por 0,033-0,048 $\mathrm{mm}$ de largura, e que depois se continua pelo conduto vaginal estreito, que se abre no oótipo. Folículos vetilínicos ovais muito pequenos, cecais, inter e extra cecais, que se estendem desde a zona faringeana até a extremidade posterior do corpo, unindo-se logo abaixo dos testículos, e depois rareando na zona intercecal. Vitelodutos acima da zona ovariana, unindo-se ao oviduto. Glândula de Mehlis não evidenciada. Canal gênito-intestinal ausente.

Aparelho genital masculino: Testiculos grandes, transversalmente elíticos, de contorno irregular, localizados no terço médio do corpo, cecais e intercecais, em número 




Loimos scitulus sp. n. Fig. 1: Total (Tipo, vista ventral).

Tagia ecuadori (Meserve, 1938) Sproston, 1946, Fig. 2: Total (n.o 30602-a, vista dorsal). 
Mem. Inst. Oswaldo Cruz, 70 (1), 1972



Loimos scitulus sp. n. Fig. 3: Aparelho genital (n. ${ }^{\circ}$ 30628-b, vista dorsal) fig. 4: Extremidade anterior (Tipo, vista ventral); fig. 5: Macroganchos opistaptor (n. ${ }^{\circ}$ 30628-h); fig. 6-a e b: Ovos (n. ${ }^{\circ} 30628-c$ e h).

bej - bulbo ejaculador; c - cirro; ds - duto seminal; dv - duto vaginal; oot - oótipo; ov - ovário; ovd - oviduto; pg - poro genital; pv - poro vaginal; ut - útero; $\mathrm{t}$ - testículo; - v - vagina; vd - viteloduto. 
aproximado de 15. O ducto seminal (vaso deferente) parte dos testículos, dirige-se cara diante até a região da bifurcação cecal e retorna novamente pelo lado contrário, onde se alarga na zona equatorial do oótipo, formando um bulbo ejaculador muscular em forma de frasco, que mede 0,099-0,129 $\mathrm{mm}$ de comprimento por 0,048 $0,066 \mathrm{~mm}$ de largura máxima, e que se continua por um tubo quitinoso fino, que representa o cirro e mede 0,180-0,231 $\mathrm{mm}$ de comprimento; terminando no poro genital.

Hibitat: Brânquias de Scolidoni terrae-novae (Rich).

Proveniência: Ilha da Marambaia, Estado do Rio de Janeiro, Brasil.

Tipo n. ${ }^{\circ} 30.628 \mathrm{a}$ e parátipos n. 30.628 $b-h$ depositados na Coleção Helmintológica do Instituto Oswaldo Cruz.

Dos dezesseis exemplares coletados, cito foram estudados, e as medidas estão baseadas em quatro.

\section{DISCUSSÃO}

Encontram-se quatro espécies no gênero Loimos MacCallum, 1917, que são: Loimos salpinggoides MacCallum, 1917; Loimos scolidoni (Mantner, 1938) Mantner, 1944; Loimos secundus (Chauhan \& Bhallerao, 1945) Chauhan \& Bhallerao, 1945; e Loimos winteri Caballero Y C. \& Bravo-Hollis, 1961. Sendo esta a primeira ocorrência de representantes deste gênero registrada no Atlântico Sul.

Loimos scitulus sp. n. agora descrita, aproxima-se de $L$. salpinggoides principalmente pela formação do proaptor, segundo Price, 1938, forma do bulbo ejaculador e cirro; de $L$. secundus pela posição do poro genital, forma do bulbo ejaculador; de $L$. winteri pela formação do opistaptor. Diferencia-se da primeira pelo tamanho do oótipo que na nova espécie é grande e facilmente evidenciado, no número de testículos, forma do ovo, formação do opistaptor e posição do poro genital; da segunda pela formação do proaptor, por possuir oótipo grande, número de testículos, formação do opistaptor e forma do ovo e da terceira na forma do proaptor, tamanho do oótipo, número de testículos e posição do poro genital. Ainda de L. scolidoni pela formação do proaptor, oótipo grande, número de testículos, posição do poro genital e formação do opistaptor.

Tagia ecuadori (Meserve, 1938) Sproston, 1946.

Os quatro exemplares por nós estudados correspondem à espécie Tagia ecuadori (Meserve, 1938) Sproston, 1946, pois tanto as suas medidas como as formas e estruturas se assemelham. Os ganchos do disco genital são em número de 14-15, apresentando a estrutura conhecida. Os folículos vitelínicos ocupam quase toda a área do corpo do animal desde o nível do disco genital até a extremidade anterior do opistaptor, chegando a penetrar ligeiramente neste, deixando apenas livre a região ocupada pelo ovário e pelos testículos. Observada cuidadosamente, a região onde Lamothe Argumedo, 1967, localizou as duas vaginas papiladas, só encontramos uma vagina muito musculosa, látero ventral, do lado esquerdo do animal (vista ventral). Nenhum dos exemplares continha ovos.

Principais medidas: Exemplares com 2,46-3,72 $\mathrm{mm}$ de comprimento por 1,08$1,160 \mathrm{~mm}$ de largura máxima. Opistaptor mede $0,72-1,10 \mathrm{~mm}$ de comprimento por 0,84-0,104 $\mathrm{mm}$ de largura e contém grampos com 0,200-0,240 $\mathrm{mm}$ de comprimento por $0,225-0,265 \mathrm{~mm}$ de largura. Ventosa oral esquerda $0,180-0,210 \mathrm{~mm}$ de compri- 
mento por $0,129-0,150 \mathrm{~mm}$ de largura, ventosa oral direita mede $0,147-0,207 \mathrm{~mm}$ de comprimento por $0,11-0,129 \mathrm{~mm}$ de largura. Disco genital com 0,090-0,105 mm de comprimento por $0,080-0,117 \mathrm{~mm}$ de largura. Distância da vagina à extremidade anterior $0,312-0,384 \mathrm{~mm}$.

Nesta oportunidade apresentamos pela primeira vez a ocorrência desta espécie na costa do Atlântico Sul.

Habitat - Brânquias de Spheroides formosus (Gunther).

Froveniência - Ilha da Marambaia, Estado do Rio de Janeiro, Brasil.

Material estudado depositado na Coleção Helmintológica do Instituto Oswaldo Cruz, sob o n. ${ }^{\circ}$ 30.602a-d.

Esta espécie é encontrada também parasitando peixes do Pacífico Norte e Sul.

\section{SUMMARY}

Monogenetic Trematodes from the south Atlantic Coast

In this paper we describe a new Mcnogenetic 'Trematode of the genus Loimos MacCallum, 1917 (Loimolinae Price, 1936; Loimoidae Bychowsky, 1957) from the gills of Scolidoni serraenovae (Rich), and we give a new record for Tagia ecuadori (Meserve, 1938)
Sproston, 1946, at the south Atlantic Ccast, with a brief description.

Loimos scitulus $\mathrm{sp}$. $\mathrm{n}$. differs from the cther species of the genus by the form of the proaptor, by the big ootipe, by the number of testicles, by the position of the genital pore, by the filament of the egg and by the form of the opistaptor. There are simmiliarities with the proaptor of Loimos salpinggoides; with the position of the genital pore of Loimos secundus; and with the opistaptor of Loimos winteri.

Although the specimen of Tagia ecuadori were found to have a muscular vagina, we identified them to this species for all the structures and measures are in the variation given for this group.

\section{AGRADECIMENTOS}

Agradecemos ao Prof. Dr. Eduardo Caballero y C. do Instituto de Biologia da Universidade do México, por nos ter emprestado um exemplar de Tagia ecuadori (Meserve, 1938) Sproston, 1946, que serviu de comparação para o nosso material.

\section{REFERÊNCIAS BIBLIOGRÁFICAS}

BYCHOWSKY, B. E., 1957, Monogenetic Trematodes. Their Sistematics and Philogeny. Akad. Nauk. SSSR, :1509. Translated from the Russian by W. J. Hargis Jr., AIBS, Washington, D.C.

CARALLERO y C. E., BRAVO-HOLLIS, M. \& GROCOTT, R. G., 1953, Helmintos de la República de Panamá. VII. Descripción de algunos tremátodos de peces marinos. An. Inst. Biol. México, 24 (1) : 97-136, 24 figs.
CABALLERO y C. E. \& BRENES. M. R. R., 1957, Helmintos de la República de Costa Rica. VI. Algunos tremátodos de peces, reptiles y mamiferos. $A n$. Inst. Biol. México, 28 (1-2) : 217-240, 10 figs.

CABALLERO y C. E. \& BRAVO-HOLLIS, M., 1961, Tremátodos de peces de águas mexicanas del Pacífico. XX. Tres especies de Monogenoidea Bychowsky, 1937. An. Inst. Biol. México, 32 (1 e 2) : 201-217, 10 figs. 
CHAUHAN, B. S., 1945, Trematodes from Indian marine fishes. Part I. Proc. 32nd. Indian Sci. Congr. Abstr. 16 (não visto) .

CHAUHAN, B. S., 1945, Trematodes from the Indian marine fishes. Part. I. On some new monogenetic trematodes of the suborder Monopisthocotylea Odhner, 1912 and Polyopisthocotylea Odhner, 1912. Proc. Indian Acad. Sc., 21 (3) : Sect. B: 129-159 (não visto).

CHAUHAN, B. S., 1959 , Studies on the Trematode Fauna of India. Part I. Subclass Monogenea. Rec. Indian Mus., 51 (2) : 113-208, 39 figs.

HARGIS, W. J. (Jr.), 1956, Monogenetic Trematodes of Gulf of Mexico Fishes. Part VIII. The superfamily Diclidophoroidea Price, 1936 (continued). Proc. Helm. Soc. Wash., 23 (1) : 5-134, 9 figs.

KORATHA, K. J., 1955, Studies on the Monogenetic Trematodes of the Texas Coast. I. Results of a survey of marine fishes at Port Aransas, with a review of Monogenea reported from the Gulf of Mexico and notes on euryhalinity, host specificity, and relationship of the Remora and the Cobaia. Inst Marino Sci., 4 (1) : 235249.

KORATHA, K. J., 1955, Studies on the Monogenetic Trematodes of the Texas Coast. II. Descriptions of species from marine fishes of Port Aransas. Inst. Marino Sci., 4 (1) : 253278, 49 figs.

LAMOTHE-ARGUMEDO, R., 1967, Monogemeos de peces. V. Redescripcion de Tagia ecuadori (Meserve, 1938) Sproston, 1946. Am. Inst. Biol. Nal. Auton. México 38, Ser. Zool. (1) : 3546, 12 figs.

MacCALLUM, G. A., 1917, Some new forms of parasitic worms. Zoopatologica 1 (2) : 43-75, 36 figs.

MANTER, H. W., 1938, Two new monogenetic Trematodes from Beaufort, North Carolina, Livro Jubilar Prof. Lauro Travassos; 293-298.

MANTER, H. W., 1944, Notes on the trematode subfamily Loimoinae (Monogenea), with a description of a new genus. J. Wash. Acad. Sci., 34 (3): 86-89, 7 figs.

MESERVE, F. G., 1938, Some Monogenetic Trematodes from the Galopos Islands and the neighboring Pacific. Allan Hancock Pacific Expeditions (Univ. S. Calif. Publ.) 2 (5) : 27-89 (não visto).

PRICE, E. W., 1938, North American monogenetic Trematodes, II. The families Mo no cotylida e, Microbothriidae, Acanthocotylidae and Udonellidae (Caspaloidea). J. Wash. Acad. Sci., 28 (3 e 4) : 109-126.

SPROSTON, N. G., 1946, A synopsis of the Monogenetic Trematodes. Trans. Zool. Soc. London, 25 (4) : 185-600, 118 figs

TRIPATHI, Y. R., 1957, Monogenetic Trematodes from fishes of India. Ind. J. Helm., 9 ( 1 e 2) : 1-149, 56 figs.

YAMAGUTI, S., 1963, Systema Helminthum, IV. Monogenea and Aspidocotylea, 699 pp., 134 pls., 898 figs. Interscience Fubl. Inc. ed., New York. 